\title{
Optimization of Cloning Conditions for High-level Production of Recombinant Mouse Interleukin-2 in Escherichia coli
}

\section{Arezou Abdi ${ }^{1}$, Mitra Hosseinpour ${ }^{1}$, Kazem Mashayekhi' ${ }^{1}$, Mohammad Javad Mousavi ${ }^{2,3}$, Seyedeh Elham Badiee Kheirabadi,1,4, and Mojtaba Sankian'}

${ }^{1}$ Immuno-Biochemistry lab, Immunology Research Center, Mashhad University of medical Sciences, Mashhad, Iran

${ }^{2}$ Department of Hematology, Faculty of Allied Medicine, Bushehr University of Medical Sciences, Bushehr, Iran

${ }^{3}$ Immunology Department, School of Medicine, Tehran University of Medical Sciences, Tehran, Iran

${ }^{4}$ M.Sc. of Immunology, Torbat Heydariyeh University of Medical Sciences, Torbat Heydariyeh, Iran

Corresponding Author: Mojtaba Sankian, PhD. Immuno-Biochemistry lab, Immunology Research Center Buali Research Institute, School of Medicine, Mashhad University of Medical Sciences, Mashhad, Iran email: sankianm@mums.ac.ir Phone: + 98513-7112610

Production and Hosting by Knowledge E

(c) Arezou Abdi et al. This article is distributed under the terms of the Creative

Commons Attribution License,

which permits unrestricted use and redistribution provided that the original author and source are credited.

Editor-in-Chief:

Dr. Alireza Rafiei

\section{Abstract}

Backgrounds and objectives: Many proteins have been expressed so far in bacterial host. Due to its simple culture conditions, having a short life cycle, and easily genetic manipulation, E.coli have been regarded as a preferable host to produce recombinant proteins, but protein cloning in bacterial host have many challenges. Therefore, we aimed to review some of these problems by an experience from mice IL-2 recombinant. Methods: cDNA synthesis was performed after RNA extraction of mouse splenocytes. PCR product purification carried out after IL-2 coding sequence amplification and was ligated into the pET-21b $(+)$ vector and transformed into the competent BL21 E.coli. Expression and purification of recombinant mouse IL-2 were done using IPTG inducer and metal affinity chromatography respectively.

Results: DNA sequencing confirmed the accuracy of the insertion process. A $23 \mathrm{kDa}$ exogenous protein was observed on the SDS-PAGE. Specificity and concentration of produced mouse recombinant IL-2 protein were confirmed by western blotting and BCA methods.

Conclusion: Recombinant IL-2 was produced in BL21 and pET-21b (+) expression system at $24^{\circ} \mathrm{C}$ in the soluble form.

Keywords: Recombinant protein; pET-21b (+); IL-2 protein; Cloning

\section{Introduction}

Despite the development of various type of simple and sophisticated various methods of protein expression and purification during the past decade, protein solubility is still a major obstacle in the cloning process. Due to the increased cloning projects and 
information obtained from previous studies showed reduction (ranging between 13\% and $23 \%$ ) of the heterologous proteins expression in E.coli and expression of some kinases, phosphatases, membrane-associated proteins and many other enzymes in insoluble form, new attempt guided towards substitution of modified expression methods that can improve the solubility of the expressed protein.(7, 8). Because protein activity demands to fold into precise three-dimensional structures (9) and functional protein should be refolded by time-consuming from inclusion bodies (10), some irregular results make the process arduous (11). Therefore, straight soluble production would be highly desirable.

Moreover, several factors should be considered to expression desired protein in the soluble form. These factors including a choice of suitable E.coli expression strain host (12), diminish the temperature (13), selection of different promoters or induction situations (14), using molecular chaperones and folding modifier in the same condition of expression (15) and uses the compatible vector to the expression host. The latest can improve expression and yield of the favorite protein consequently. A vector can assert solubility and/or fold in one of three ways: First, facilitate binding to a protein that is highly soluble in itself, e.g. glutathione-S-transferase (GST), thioredoxin (Trx), $\mathrm{N}$ utilization substance A (NusA). Second Providing conditions for better linkage to the enzyme responsible for breaking disulfide bonds formation (e.g. thioredoxin, DsbA, DsbC) (16), and finally simplification the translocation of protein into the peri-plasmic space by forming signal sequence (17).

Mouse IL-2 (also known as TCGF) is a 17 kDa factor produced mainly by a specific subset of $\mathrm{T}$ cells. The important role of $\mathrm{T}$ cells in the immunity system consisting of cell cycle progression, clonal expansion, and proliferation of activated T cells as well as NK cells, LAK cells, monocytes and B cells growth factor secretion. Simplify the soluble expression of this protein can make the process easy for further studies on the utility of this product particularly in the development of diagnostic methods such as ELISA and promote proliferation of mouse $\mathrm{T}$ lymphocytes in culture. Therefore, the aim of this study was to clone the mouse IL-2 for optimization of eukaryotic proteins cloning condition.

\section{Material and Methods}

\subsection{RNA isolation and RT- PCR}

Total RNA was isolated from mouse splenocytes using RNA extraction kit (Pars Tous, Iran, Cat no. A101231). The cDNA was constructed by using the reverse transcription 
reaction mixture containing oligo-dT primers and prepared Master Mix following the standard protocol (Pars Tous, Iran, Cat no. A101161).

The amplification of coding region of IL2 was done using two IL-2 gene-specific primers designed base on the sequence of mature mouse IL-2 available in the Unipart database. These primers contained EcoRl and Xhol restriction enzyme sites without the signal peptide. The designed primers sequences were: ECoR1-Forward 5'GATCCGAATTCGGCACCCACTTCAAGCTCCACT3' and Xhol-Reverse 5'TGGTGCTCGAGTTGAGGGCTTGTTG AGATGATGC3'

A standard Polymerase Chain Reaction (PCR) protocol was conducted as follows: DNA template (10 ng) Gene-specific primers (10 pmol/ $/$ l), 10x PCR buffer, dNTP (10 mM), MgCl2 (25 mM) and KlenTaq DNA polymerase (5 U/Nl) (Pars Tous, Iran, Cat no. C101121). The PCR amplification conditions was initial denaturation at $94^{\circ} \mathrm{C}$ for $3 \mathrm{~min}, 28$ cycles of denaturation at $94^{\circ} \mathrm{C}$ for $45 \mathrm{~s}$, primer annealing at $58^{\circ} \mathrm{C}$ for $30 \mathrm{~s}$ and DNA extension at $72^{\circ} \mathrm{C}$ for 30s. Finally, the reaction was carried out at $72^{\circ} \mathrm{C}$ for $3 \mathrm{~min}$.

\subsection{Cloning and expression}

A 447 bp PCR fragment from IL2 gene was assessed by agarose gel electrophoresis. The PCR product was extracted from agarose gel using DNA extraction kit (Pars Tous, Iran, Cat no. A101221), and after digestion with EcoRI and Xhol, than digested product was ligated into $\mathrm{pET}$-21b (+) vector with DNA ligase according manufacture instructions (Thermo Fisher Scientific, USA).

The recombinant plasmid transformed into competent TOP10 E.coli cells as previously describe (18).. Inserted colonies were screened for ampicillin resistance. After ensuring the accuracy of the sequence of inserted plasmid (Macro gene, Germany), transformation into the competent $E$.coli $\mathrm{BL} 21$ cells was performed and induced by $0.2 \mathrm{mM}$ Isopropyl $\beta$ - Dthiogalactoside (IPTG) and incubated for 18 hours at $24^{\circ} \mathrm{C}$. The bacteria were harvested by centrifugation at $13400 \mathrm{rpm}$ for $1 \mathrm{~min}$. After re-suspension in the lysis buffer (Potassium Phosphate buffers (50 mM, pH 7.8), Glycerol 10\%, Triton x-100 0.5\%, Nacl $200 \mathrm{mM}$ ) and sonication, protein expression was confirmed by loading supernatant and cell pellet on the SDS-PAGE. Expressed recombinant protein was employed to a ( $\mathrm{Ni}$ IDA) chromatography column in order to purify histidine-tagged proteins according to the manufacturer's instructions (Pars Tous, Iran, Cat no. A101271), in briefly, 10ml bacteria lysate was passed through chromatography column. Then, column was washed with wash buffer I (Potassium Phosphate buffers (50 mM, pH 7.8), Nacl 200 mM) and wash buffer II (wash I, 20 mM Imidazole) respectively. Finally, protein was eluted with elution 
buffer (wash I, $250 \mathrm{mM}$ Imidazole). The purified protein was dialyzed in PBS buffer at $4^{\circ} \mathrm{C}$ for 24 hours.

\subsection{Western blot analysis}

After running the expressed purified recombinant mouse IL-2 protein on the $\% 12.5$ electrophoresis gel, they were blotted to polyvinylidene difluoride (PVDF) membrane and blocked with BSA $2 \%$ overnight at $4^{\circ} \mathrm{C}$. After washing, lanes were incubated with 1:500 diluted biotinylated anti-mouse IL-2 (Ebioscience, Vienna, Austria) for 2 hours at room temperature. Then the membranes were incubated with 1:20000 diluted horseradish peroxidase-streptavidin (HRP) conjugate (Bio-Rad, CA, USA) 1 hour at RT. In the last step, the specific reaction was detected by Chemiluminescent substrate (Pars Tous, Iran, Cat no. B111420).

\subsection{Protein concentration quantification}

Bicinchoninic Acid (BCA) protein assay method was used in order to quantify the concentration of recombinant protein. All samples and reagents were prepared according manufacture instructions (Pars Tous, Iran, Cat no. A101251) and tested in a duplicate way. The optical density of samples was detected at $562 \mathrm{~nm}$ and final concentration obtained by comparing the result with the standard curve.

\section{Results}

\subsection{Amplification, cloning and sequence of cDNA coding mouse IL-2}

As reported in Unipart database the length of mouse IL-2 cDNA is 510 bp (accession number: PO4351). After amplification of mouse IL-2, we were able to obtain a fragment about $447 \mathrm{bp}$ (Figure 1). The fragment was inserted into the pET-21b (+) expression vector; one clone was confirmed using PCR with T7 primers. The PCR product showed a fragment of approximately $687 \mathrm{bp}$ (Figure 2). Finally, aligning the nucleotide sequences with the sequence of mouse IL-2 deposited from GenBank revealed complete homology (Figure 3). 


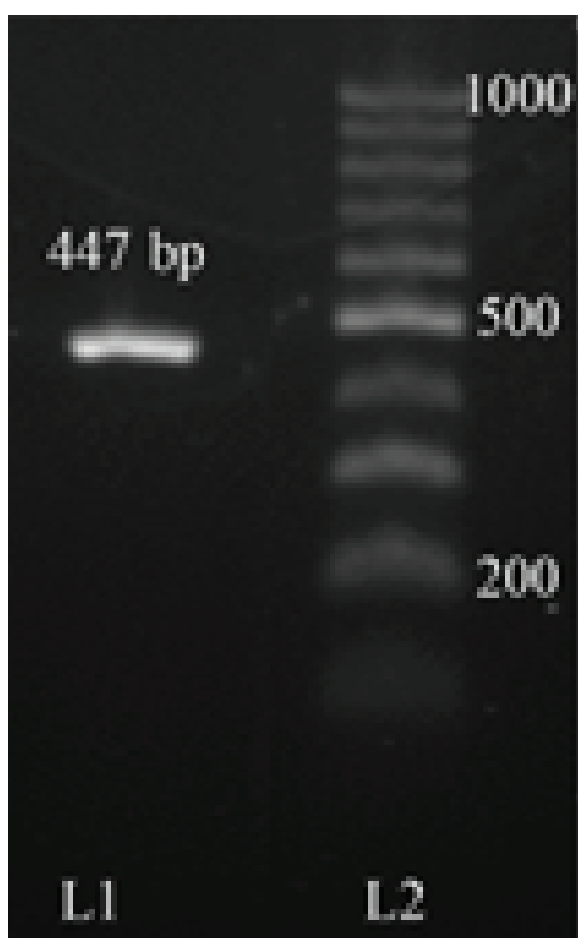

Figure 1: Agarose gel electrophoresis of PCR product after amplification of the mouse IL-2 coding region using the specific primers. Lane 1: PCR product of $447 \mathrm{bp}$ mouse IL-2. Lane 2: DNA marker (100 bp, Fermentas).

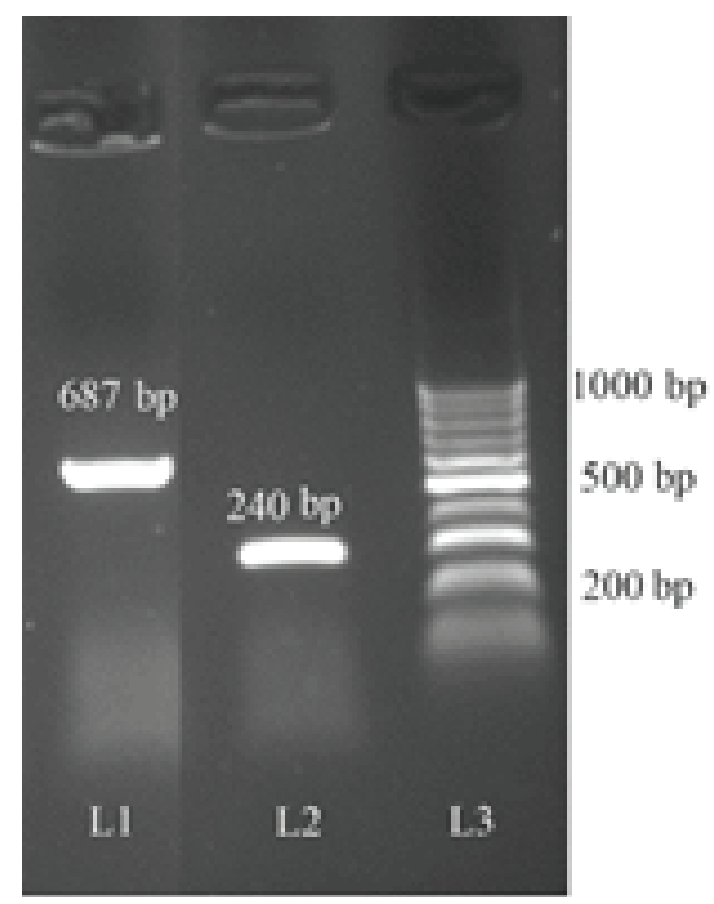

Figure 2: Colony-PCR screening with T7 universal primers. Lane 1: 687 bp recombinant plasmid, Lane 2: plasmid without insert, Lane 3: DNA marker (100 bp, Fermentas). 


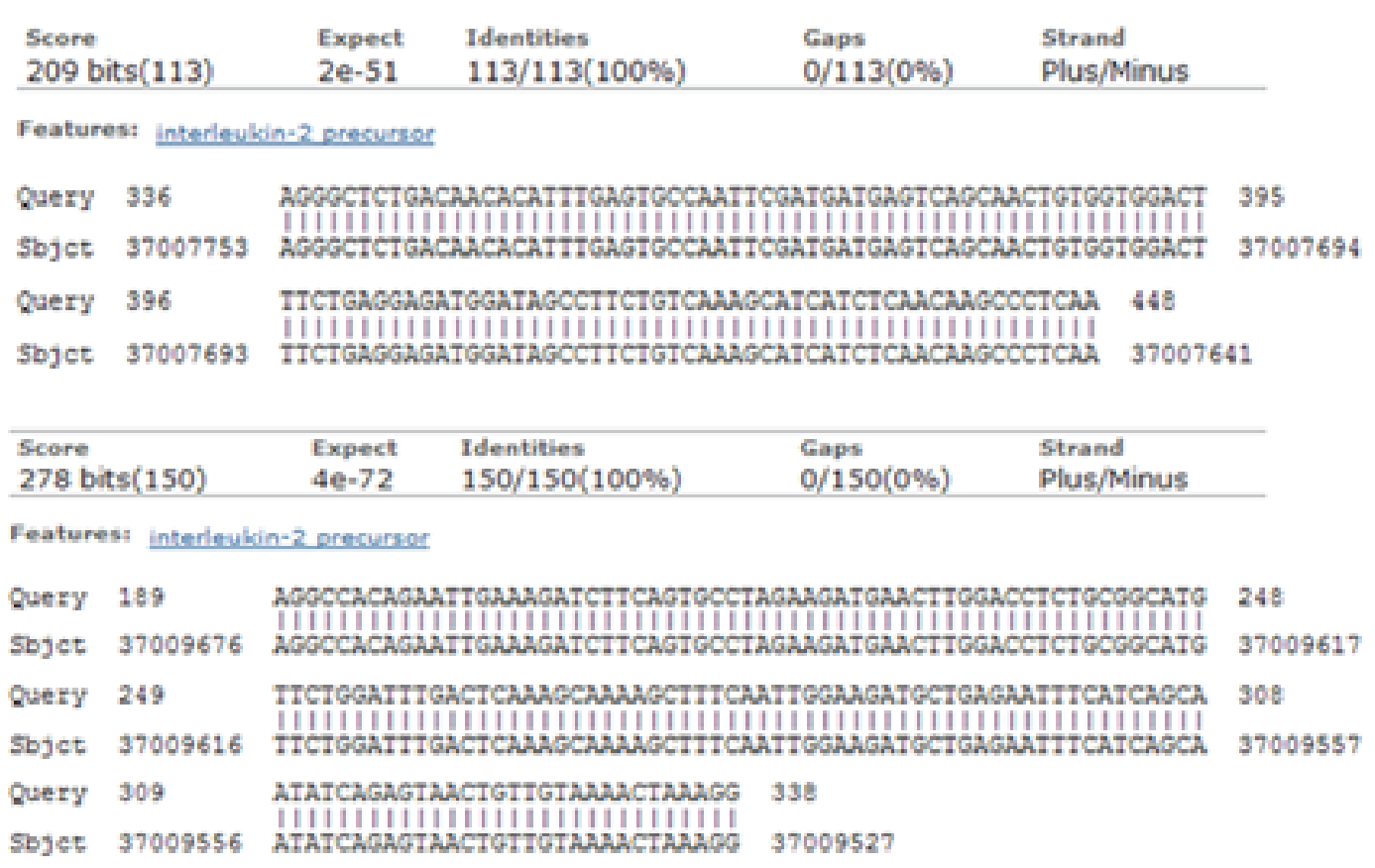

Figure 3: Aligning the nucleotide sequences with the sequence of mouse IL-2 from GenBank.

\subsection{Expression and purification}

The recombinant plasmid was transformed into the expression host E.coli strain BL21. After IPTG induction for 18h, 23 kDa recombinant mouse IL-2 was expressed on SDSPAGE electrophoresis gel in the soluble form (Figure 4). For the purification of the protein, the cells were sonicated on ice and employed to affinity chromatography (Figure 5) as well as purified using dialyzes.

\subsection{Immunoblotting}

Immunoblotting of the mouse IL-2 protein with 1:500 diluted biotinylated anti-mouse IL2 was done and results showed that our protein was expressed as a $23 \mathrm{kDa}$ fragment (Figure 6). Also, we noticed that IL-2 folded as a $46 \mathrm{kDa}$ dimer protein.

\section{Discussion}

It is ideal overcoming the problem of non-soluble expression of various proteins and simplification the process of production of recombinant proteins. Checking the solubility of desired protein with available software would be useful before cloning the target gene into the particular vector. Despite obtained information based on available software that 


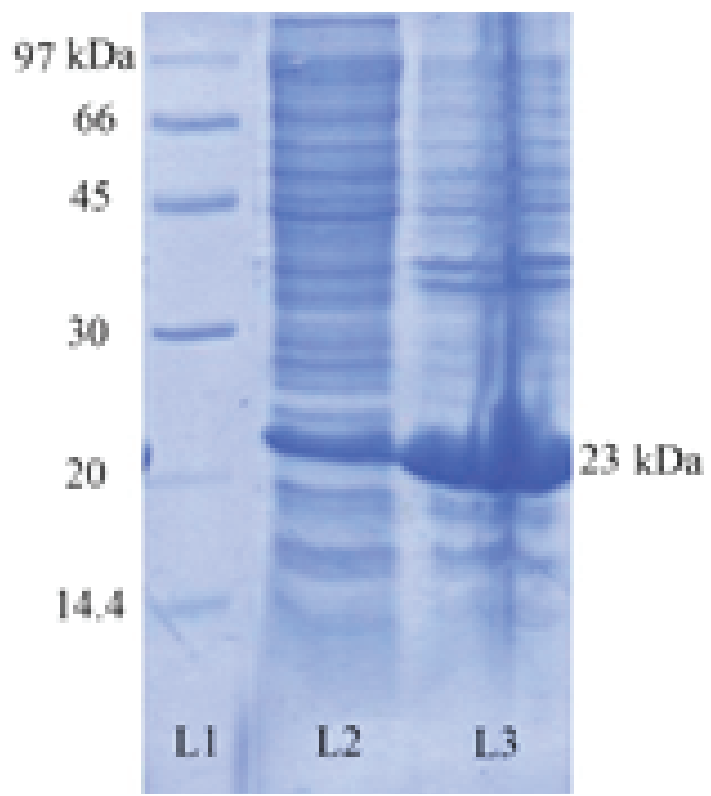

Figure 4: SDS-PAGE for analyzing of Mouse IL-2 expression with Coomassie blue staining. Lane 1: The Protein size marker. Lane 2: Expressed IL-2 protein in the supernatant. Lane 3: Expressed IL-2 protein (23 $\mathrm{kDa}$ ) in the precipitate.

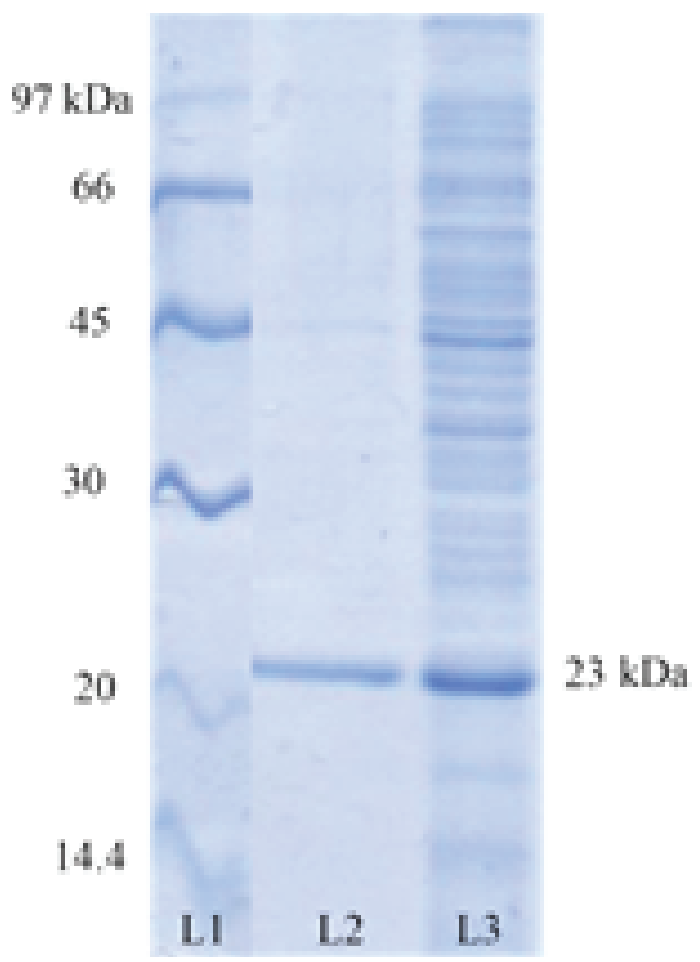

Figure 5: SDS-PAGE for analyzing the purity of mouse IL-2 protein after Nickel affinity chromatography. Lane1: The Protein size marker. Lane 2: Purified protein in 23 kDa. Lane 3: expressed recombinant mouse II-2 befor purification.

showed the low percentage of soluble expression of mouse IL-2, in the current study, we have produced recombinant mouse IL-2 in the soluble form using pET-21b (+) vector in BL21 (DE3) E. coli strain. 


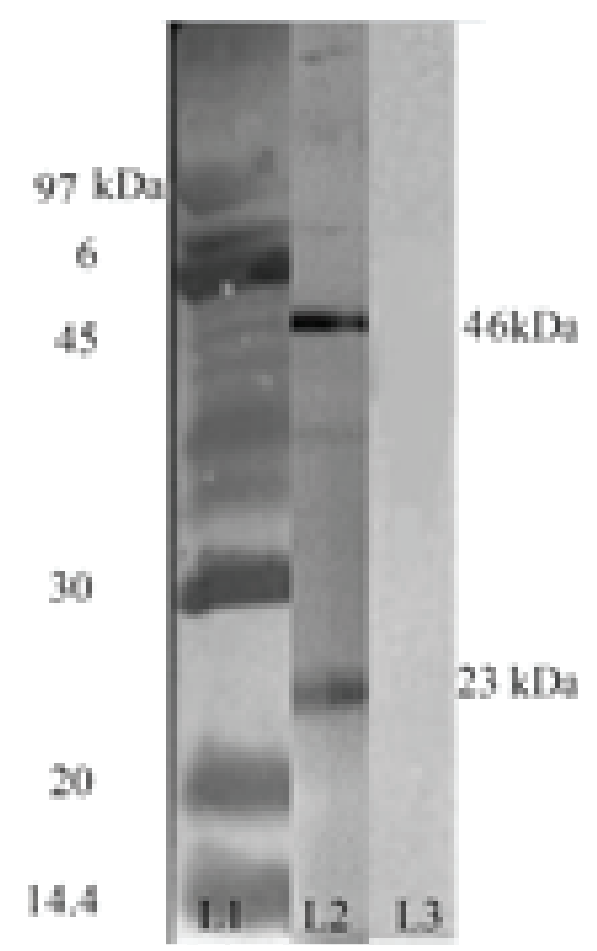

Figure 6: Western blotting of purified recombinant mouse IL-2. Lane 1: Low molecular weight protein size marker. Lane 2: Purified recombinant protein. Lane 3: Negative control BL21.

One of the most important steps in the process of cloning is choosing the best vector can provide our interests in the process of cloning. Vector selection depends on the promoter with which the gene of interest is to be cloned and different fusion 'tags' which influence solubilization of recombinant protein in E.coli host (19). In this study, we used pET-21b (+) vector and the reason for choosing mentioned vector is eliminating the fusion tag removal step because of the existence of a smaller tag, a 6-Histidine tag (His-tag) in this vector, in comparison with other pET vectors such as pET-32a $\left(^{+}\right)$which is previously used in mouse IL-2 cloning process (20). Since pET-32a (+) contain a long fusion tag which adds 109-aa, this large size can interfere with the activity of recombinant protein if the tag is not cleaved properly. In the other hand, appropriate enzyme sites must be designed into the vector in order to precise cleavage and complete removal extra amino acid fused with recombinant protein. In addition due to the interference of long tags such as thioredoxin with the structure and the function of desired recombinant protein, therefore it must be removed after the expression and purification process $(20,21)$.

In the recent study, we observed the higher molecular weight of produced recombinant protein than we expected. This experience also has been implied by Yan et al. The reason for this phenomenon is related to various protein conformation and formation of a disulfide bond between protein monomers resulting in formation protein in dimer form. Moreover, the existence of His-tag at the end of a protein can have an effect on the 
conformational structure of target protein resulting in changing the mobility of protein on the SDS-PAGE gel electrophoresis (22).

In our experiment, we have expressed recombinant mouse IL-2 in the soluble form by optimizing temperature in $24^{\circ} \mathrm{C}$. According to the previous study the optimum temperature for induction of recombinant proteins is $37^{\circ} \mathrm{C}(23)$. However, inclusion body formation is facilitated in $37^{\circ} \mathrm{C}$. In addition, the temperature can be an effective factor in the expression rate of recombinant protein production as well as appropriate folding of the recombinant protein (24). Optimization the time incubation and decreasing temperature to lower $\left(15-20^{\circ} \mathrm{C}\right)$ can affect the growth of bacteria, rate of protein production and reduction of aggregation of intended protein $(25,26)$.

Using IPTG to induce lac repressor-regulated promoters can influence the cell mass as well as yield of desired protein (27). Protein expression can be regulated by optimizing the concentration of IPTG. Some proteins need to be induced slowly with lower IPTG concentrations while others, higher amounts of IPTG is desired (28). Since establishing optimum concentration will gain empirically by varying the IPTG concentration between 0.01 and $5 \mathrm{~mm}$, we set up $0.2 \mathrm{mM}$ concentration of IPTG achieved by examining different concentration of IPTG.

\section{Conclusion}

We facilitated cloning and expression of mouse recombinant IL-2 using by vector pET$21 \mathrm{~b}(+)$ in the soluble form. This cytokine was expressed at $24^{\circ} \mathrm{C}$ in large scale without any refolding and it is suitable for commercial aims. In order to uses of this cytokine for clinical use, it is better that it cloned in eukaryotic hosts such as pichia pastoris.

\section{Acknowledgment}

This study "was supported by Grant 930575 from the Research Administration Department of Mashhad University of Medical Sciences, Mashhad, Iran.

\section{Author Contributions}

AA performed experiments and prepared the draft of the paper. $\mathrm{MH}$ collaborated on experiments and manuscript preparation. KM developed the main idea, and performed the statistical analysis. MJM assessed the obtained data and edit the manuscript critically. SEBK designed the work, and read the manuscript. MS developed the main 
idea, read and edit the manuscript critically. All authors have read and approved the manuscript.

\section{References}

[1] Ma A. Pleiotropic functions of IL-15 in innate and adaptive immunity. Mod Asp Immunobiol. 2000;1:102-4.

[2] Carson WE, Fehniger TA, Haldar S, Eckhert K, Lindemann MJ, Lai C-F, et al. A potential role for interleukin-15 in the regulation of human natural killer cell survival. J Clin Invest. 1997;99(5):937.

[3] Giri JG, Ahdieh M, Eisenman J, Shanebeck K, Grabstein K, Kumaki S, et al. Utilization of the beta and gamma chains of the IL-2 receptor by the novel cytokine IL-15. EMBO J. 1994;13(12):2822.

[4] Giri JG, Kumaki S, Ahdieh M, Friend DJ, Loomis A, Shanebeck K, et al. Identification and cloning of a novel IL-15 binding protein that is structurally related to the alpha chain of the IL-2 receptor. EMBO J. 1995;14(15):3654.

[5] Khatri VP, Fehniger TA, Baiocchi RA, Yu F, Shah MH, Schiller DS, et al. Ultra low dose interleukin-2 therapy promotes a type 1 cytokine profile in vivo in patients with AIDS and AIDS-associated malignancies. J Clin Invest. 1998;101(6):1373.

[6] Blackman MA, Tigges MA, Minie ME, Koshland ME. A model system for peptide hormone action in differentiation: interleukin 2 induces a $B$ lymphoma to transcribe the J chain gene. Cell. 1986;47(4):609-17.

[7] Chambers SP, Austen DA, Fulghum JR, Kim WM. High-throughput screening for soluble recombinant expressed kinases in Escherichia coli and insect cells. Protein Expr Purif. 2004;36(1):40-7.

[8] Marblestone JG, Edavettal SC, Lim Y, Lim P, Zuo X, Butt TR. Comparison of SUMO fusion technology with traditional gene fusion systems: enhanced expression and solubility with SUMO. Protein Sci. 2006;15(1):182-9.

[9] Villaverde A, Carrió MM. Protein aggregation in recombinant bacteria: biological role of inclusion bodies. Biotechnol Lett. 2003;25(17):1385-95.

[10] Middelberg AP. Preparative protein refolding. Trends Biotechnol. 2002;20(10):43743.

[11] Clark EDB. Protein refolding for industrial processes. Curr Opin Biotechnol. 2001;12(2):202-7. 
[12] Miroux B, Walker JE. Over-production of proteins inEscherichia coli: mutant hosts that allow synthesis of some membrane proteins and globular proteins at high levels. J Mol Biol. 1996;260(3):289-98.

[13] Hammarström M, Hellgren N, van den Berg S, Berglund H, Härd T. Rapid screening for improved solubility of small human proteins produced as fusion proteins in Escherichia coli. Protein Sci. 2002;11(2):313-21.

[14] Qing G, Ma L-C, Khorchid A, Swapna G, Mal TK, Takayama MM, et al. Coldshock induced high-yield protein production in Escherichia coli. Nat Biotechnol. 2004;22(7):877-82.

[15] de Marco A, De Marco V. Bacteria co-transformed with recombinant proteins and chaperones cloned in independent plasmids are suitable for expression tuning. J Biotechnol. 2004;109(1):45-52.

[16] Terpe K. Overview of tag protein fusions: from molecular and biochemical fundamentals to commercial systems. Appl Microbiol Biotechnol. 2003;60(5):523-33.

[17] Du Plessis DJ, Nouwen N, Driessen AJ. The sec translocase. Biochimica et biophysica acta. 2011;1808(3):851-65.

[18] Chang AY, Chau V, Landas JA, Pang Y. Preparation of calcium competent Escherichia coli and heat-shock transformation. JEMI Methods. 2017;1:22-5.

[19] Gopal GJ, Kumar A. Strategies for the production of recombinant protein in Escherichia coli. Protein J. 2013;32(6):419-25.

[20] Novy R, Berg J, Yaeger K, Mierendorf R. pET TRX fusion system for increased solubility of proteins expressed in E. coli. Innovations. 1995;3:7-9.

[21] LaVallie E, DiBlasio E, Kovacic S, Grant KL, Schendel PF, McCoy JM. A thioredoxin gene fusion expression system that circumvents inclusion body formation in the $\mathrm{E}$. coli cytoplasm. Biotechnol J. 1993;11:187-93.

[22] Yan WK, Goette M, Hofmann G, Zaror I, Sim J. High-level soluble expression, purification and characterization of active human midkine from Escherichia coli. Protein Expr Purif. 2010;70(2):270-6.

[23] Mir Mohammad Sadeghi H, Rabbani M, Rismani E, Moazen F, Khodabakhsh F, Dormiani K, et al. Optimization of the expression of reteplase in Escherichia coli. Res Pharm Sci. 2011;6(2):87-92.

[24] Li R-Y, Cheng C-Y. Investigation of inclusion body formation in recombinant Escherichia coli with a bioimaging system. J Biosci Bioeng. 2009;107(5):512-5.

[25] Vera A, González-Montalbán N, Arís A, Villaverde A. The conformational quality of insoluble recombinant proteins is enhanced at low growth temperatures. Biotechnol Bioeng. 2007;96(6):1101-6. 
[26] Schein $\mathrm{CH}$. Production of soluble recombinant proteins in bacteria. Nat Biotechnol. 1989;7(11):1141-9.

[27] Grabski A, Mehler M, Drott D. The overnight express autoinduction system: high-density cell growth and protein expression while you sleep. Nat Methods. 2005;2(3):233-5.

[28] Sambrook J, Russell D. Molecular Cloning, A Laboratory Manual. 3rd Edition, Vol. 1, Cold Spring Harbor laboratory Press. Cold Spring Harbor, New York; 2001. 